\title{
Tierversuche, Studienregister und Patientensicherheit
}

\author{
Derzeit wird das Schweizerische Studienregister für Tierversuche revidiert. Auch \\ der in die Vernehmlassung geschickte Entwurf enthält zahlreiche Elemente, die \\ gemäss heutigen forschungsethischen Grundsätzen nicht mehr zu verantworten \\ sind. Unabhängig davon, ob ein medizinisch relevantes Studienresultat aus ei- \\ nem Versuch am Menschen oder am Tier stammt, kann die Nichtveröffentlichung \\ schwere Folgen haben.
}

Markus Deutsch

Für den Vorstand der Ärztinnen und Ärzte für Tierschutz in der Medizin
Korrespondenz:

Dr. med. Markus Deutsch

Facharzt für

Innere Medizin FMH

Dürntnerstrasse 20

CH-8340 Hinwil

Tel. 0449374240

Fax 0449374241

m.deutsch@gmx.ch

www.aerztefuertierschutz.ch
In diesen Monaten läuft die Diskussion über die Revision der Tierversuchsstatistik des Bundesamtes für Veterinärwesen BVET zum neuen elektronischen Informationssystem für Tierversuche (E-Tierversuche). Hierdurch steht auch die Frage zur Diskussion, wer alles Zugriff haben soll auf diese Daten (4. Abschnitt, Art. 11 und 12, Zugriff auf Stammdaten und andere Daten). Im unterbreiteten Entwurf hat niemand $\mathrm{Zu}$ griff auf diese Daten ausser den beteiligten Behörden und den Forschern selbst, aber auch diese nur auf die selbst eingegebenen Daten!

In der Schweizerischen Ärztezeitung [1, 2] wie auch in zahlreichen internationalen renommierten Journals [3-7] ist in den letzten Jahren immer wieder gefordert worden, dass geplante Studien und Studienresultate in frei zugänglichen Studienregistern zu registrieren sind. Dies ist heute unter der internetbasierten Datenbank www.clinicaltrials.gov umgesetzt. Ohne Registrierung vor Studienbeginn werden Arbeiten von vielen wichtigen Journals wie beispielsweise dem New England Medical Journal (NEMJ) gar nicht mehr angenommen.

Die Gründe für diese international abgestützte Forderung nach Transparenz im Studienwesen sind schwerwiegend:

\section{Nichtpublikation von unerwünschten Resultaten kann zu falschen Behandlungs- richtlinien führen}

So hält Burnand in der SÄZ fest: «Der Entscheid, die Ergebnisse einer klinischen Studie nicht zu veröffentlichen oder bloss eine Auswahl von Resultaten bekannt zu geben, kann fatale Konsequenzen nach sich ziehen. Das zeigt das jüngste Beispiel einer Studie zur Behandlung der Depression bei Kindern und Jugendlichen mit selektiven Serotonin-Wiederaufnahmehemmern (SSRI).» [1]

Weiter: «Bei diesem Vergleich gaben die unveröffentlichten Ergebnisse den Ausschlag (dass die Risiken der Behandlung weit grösser waren als der Nutzen), denn gemäss den veröffentlichten Studien war der Nutzen grösser als die Risiken (häufigere Suizidversuche bei der Behandlung mit SSRI).»

Die gleiche Meinung vertritt Steinbrook im New England Medical Journal: «When serious questions, such as those about the relation between selective serotonin-reuptake-inhibitor antidepressants and the risk of suicide in children, are raised, there is no substitute for getting all the data out and examining them critically.» [3]

Diese Forderung nach mehr Transparenz ist auch von der Politik aufgenommen worden. Ständerat Hans Altherr, Nationalrat FDP, reichte am 16. Dezember 2008 eine Motion ein mit der Forderung, dass das Schweizerische Heilmittelinstitut Swissmedic grundsätzlich zur Transparenz zu verpflichten sei.

\section{In Anbetracht des Risikos für} den Probanden ist die Nichtveröffentlichung von Resultaten ethisch inakzeptabel

Wieder Burnand: «Denn die Patienten, die sich zur Teilnahme an einem klinischen Versuch bereit erklärt und die damit verbundenen Risiken auf sich genommen haben, erwarten zumindest, dass diese Studie nützliche Erkenntnisse für die Forschung erbringt.» [1]

Gleich sieht es Drazen im NEJM: «[...] their participation in a clinical trial should result in generalizable knowledge that will be available to future patients and investigators to improve patient care. This can happen only when appropriate details of the clinical trial are made available to the public [...].» [7]

Es ist somit zwingend, dass alle Studienresultate veröffentlicht werden, und zwar unabhängig davon, ob das Ergebnis den Wünschen des Untersuchers und Sponsors entspricht oder nicht.

\section{Doppelspurigkeiten könnten verhindert werden}

Nochmals Burnand: «Dadurch liesse sich die klinische Forschung besser planen und zudem könnten Prioritäten festgelegt und Doppelspurigkeiten von Studien vermieden werden.» [1] 
Für Studienresultate aus Tierversuchen gelten aber ohne Einschränkung genau die gleichen Vorbehalte wie für klinische Studien, wenn sie nicht in Studienregistern erfasst werden!

\section{Erforderliche Tierversuchszahlen könnten verglichen und somit reduziert werden}

Wenn Tierversuchskommissionen Versuchsanträge begutachten und abzuschätzen versuchen, ob ein Versuchsziel mit Alternativmethoden oder zumindest einer kleineren Versuchstierzahl durchgeführt werden könnte, so ist ihnen der Vergleich mit anderen aktuellen Versuchen heute durch das Amtsgeheimnis verwehrt. Aber auch Forscher selbst könnten sich über die Verwendung von Alternativmethoden und Tierversuchszahlen in aktuellen Projekten in dieser Datenbank informieren.

Unabhängig davon, ob ein medizinisch relevantes Studienresultat aus einem Versuch am Menschen oder am Tier kommt, kann die Nichtveröffentlichung schwere Folgen haben. An Tierversuche schliessen sich oft Studien am Menschen an. Mensch und Tier haben ein moralisches Recht darauf, dass die Erkenntnisse aus den Risiken und Leiden, die sie auf sich genommen haben, uneingeschränkt der Wissenschaft zur Verfügung gestellt werden

Auch im Bereich der Tierversuche liessen sich Doppelspurigkeiten durch ein öffentliches Studienregister besser vermeiden.

So lange wir davon ausgehen, dass Tierversuche für die medizinische Forschung unabdingbar sind und der «Verbrauch» von 726392 Tieren pro Jahr (2007) unverzichtbar ist, so lange müssen wir auch davon ausgehen, dass die Nichtpublikation von Tierversuchsergebnissen schwere Folgen für den Menschen haben kann, genauso wie bei den klinischen Versuchen.

Aus diesem Grunde ist die obligatorische Publikation von allen geplanten Tierversuchen genauso unverzichtbar wie bei klinischen Versuchen. Es geht nicht «nur» um Tierschutz, es genauso sehr um die Patientensicherheit!

Neben diesen schwerwiegenden Gründen kommen aber auch noch weitere hinzu:

\section{Öffentlichkeitsgesetz (Recht des Bürgers auf Transparenz in der Verwaltung)}

Am 1. Juli 2006 ist das Bundesgesetz über das Öffentlichkeitsprinzip der Verwaltung in Kraft getreten. Öffentliche Dokumente sind damit für jedermann zugänglich, soweit nicht andere schutzwürdige Interessen dem entgegenstehen.

Der Bürger hat aus verschiedenen Gründen ein Recht, über die Tierversuche in der Schweiz informiert zu werden:

1. Viele Tierversuche werden mit öffentlichen Mitteln finanziert (Schweizerischer Nationalfonds, Universitäten usw.), also letztlich mit Steuergeldern. Also hat der Bürger auch ein Recht darauf, zu erfahren, was mit seinen Geldern passiert.

2. Wie viele und wie schwerwiegende Tierversuche akzeptabel sind, wird letztlich von der Gesellschaft als Ganzes bestimmt, mittels Abstimmungen und Gesetzen. Der Bürger kann aber nicht über etwas abstimmen, das er nicht sehen darf. Der Bürger muss deshalb Einsicht haben in die heutigen Tierversuche, um sich überhaupt selbst eine Meinung bilden zu können.

\section{Zusammenfassung}

Der aktuelle Entwurf für die Tierversuchsstatistik ist somit aus wissenschaftlichen, ethischen und gesellschaftspolitischen Gründen vollständig inakzeptabel.

Er enthält viele Elemente, die selbst in Forscherkreisen heutzutage als obsolet und unverantwortbar gelten:

- Es gibt keine Pflicht, alle geplanten Studien zu erfassen und zu publizieren.

- Es gibt keine Pflicht, die Resultate der Studien zu publizieren.

Aber nicht einmal die minimalsten heutigen forschungsethischen Prinzipien werden berücksichtigt:

- Nicht einmal Forscher selbst dürfen sich ein Bild machen, ob ähnliche Projekte bereits an anderen Instituten geplant sind!

- Nicht einmal Tierversuchskommissionsmitglieder, die von Amtes wegen über die Notwendigkeit eines Tierversuchsprojekts zu entscheiden haben, dürfen nachschauen, ob ein Versuch anderswo geplant ist oder allenfalls sogar schon läuft!

Die Ärzteschaft hätte also allen Grund, sich für ein wissenschaftlich, ethisch und gesellschaftspolitisch verantwortbares Tierversuchsinformationssystem einzusetzen.

\section{Literatur}

1 Burnand B, Rège Walther M. Registrierung und Veröffentlichung klinischer Studien. Schweiz Ärztezeitung. 2005;86(8): 482-3.

2 SAMW. Aufruf zur Erfassung klinischer Studien in einem öffentlich zugänglichen Register. Schweiz Ärztezeitung. 2005;86(7):396.

3 Steinbrook R. Public registration of clinical trials. N Engl J Med. 2004;351(4):315-7.

4 De Angelis C, Drazen JM, Frizelle FA, et al. Clinical trial registration: a statement from the International Committee of Medical Journal Editors. N Engl J Med. 2004;351(12):1250-1.

5 De Angelis C, Drazen JM, Frizelle FA, et al. Is this clinical trial fully registered? - A statement from the International Committee of Medical Journal Editors. N Engl J Med. 2005;352(23):2436-8.

6 Zarin DA, Tse T, Ide NC. Trial registration at ClinicalTrials.gov between May and October 2005. N Engl J Med. 2005;353(26):2779-87

7 Drazen JM, Wood AJ. Trial registration report card. N Engl J Med. 2005;353(26):2809-11. 